\title{
VECTOR LATTICES OF SELF-ADJOINT OPERATORS
}

\author{
BY \\ DAVID M. TOPPING $\left({ }^{1}\right)$
}

1. Introduction. The relations between order and commutativity in operator algebras have been studied by a number of authors [7], [8], [10], [11]. Results in this area usually begin with an order assumption and end with a commutative conclusion. The methods used have tended to refer to $C^{*}$-algebras, while, in reality, the "commutativity-from-order" theme has little to do with such matters. The proper approach, as we propose to show, is via a kind of "local" study of the linear space and absolute value structure of the space $S$ of all self-adjoint (s.-a.) operators on a Hilbert space. Specifically, we show that the commutative subsets of $S$ are completely determined by the linear structure of $S$ together with the absolute value operation. This is no longer true if we replace the words "absolute value operation" by "partial order" (the usual one for s.-a. operators).

Locally in $S$, there is a special kind of vector lattice structure which we believe is the single underlying factor in all situations where commutativity follows from order considerations. We venture to predict that any future inferences of this sort can be rapidly disposed of via our special structure and a few elementary manipulations. To bear this out, we generalize and thus deduce the existing results in this fashion.

Finally, we raise the same questions for s.-a. measurable operators and for the s.-a. part of the regular ring of a finite $A W^{*}$-algebra. The finality achieved in the bounded case is not as yet forthcoming here; nevertheless, we do manage to reproduce substantial portions of our results.

The main results of this paper were announced in [15].

2. The absolute value and related operations. If $a$ is a s.-a. operator (bounded, except in $\S 8$ ), we set $|a|=\left(a^{2}\right)^{1 / 2}$ and define

$$
\begin{aligned}
a \vee b & =\frac{1}{2}(a+b+|a-b|), \\
a \wedge b & =\frac{1}{2}(a+b-|a-b|), \\
a^{+} & =\frac{1}{2}(|a|+a) \text { and } a^{-}=\frac{1}{2}(|a|-a) .
\end{aligned}
$$

We summarize a number of simple properties of these operations in

LEMmA 1. (1) $a \wedge b=$ a and $a \bigvee b=b$ are equivalent ways of expressing $a \leqq b$; (2) $a+b=a \bigvee b+a \wedge b$ (Dedekind's Law); (3) $|a-b|=a \bigvee b-a \wedge b$; (4)

Received by the editors May 25, 1962 and, in revised form, August 6, 1962.

( $)$ NATO Postdoctoral Fellow. 
$a \bigvee b=-(-a \wedge-b)$ and $a \wedge b=b \wedge a ;(5)(a \bigvee b)+c=(a+c) \bigvee(b+c)$ and dually for $\bigwedge$; (6) $a=a^{+}-a^{-},|a|=a^{+}+a^{-}$and $a^{+} \wedge a^{-}=0$; (7) $(a-b)^{+}$ $+a \wedge b=a$ and $(a-b)^{-}+a \wedge b=b ;(8)|\alpha a|=|\alpha| \cdot|a|$ for $\alpha$ real, $(\alpha a)^{+}$ $=\alpha a^{+}$and $(\alpha a)^{-}=\alpha a^{-}$for $\alpha \geqq 0$, while $(\alpha a)^{+}=-\alpha a^{+}$and $(\alpha a)^{-}=-\alpha a^{-}$ for $\alpha<0$; (9) $-|a| \leqq a \leqq|a| ; 0, a \leqq a^{+}=a \bigvee 0 ; 0,-a \leqq a^{-}=(-a) \bigvee 0$ and $a \wedge b \leqq a, b \leqq a \bigvee b ;(10)\|(|a|)\|=\|a\|$ and $\|a\| \leqq\|b\|$ if $0 \leqq a \leqq b$.

Verification of these facts is straightforward and is left to the reader.

Two s.-a. operators $a$ and $b$ are disjoint if $a \wedge b=0$. If this happens, then $a, b \geqq 0$ (by (9) above). Concerning this notion we have

LEMMA 2. For two s.-a. operators $a, b \geqq 0$ the following are equivalent:

(1) $a$ and $b$ are disjoint;

(2) $a b=-b a$;

(3) $a b=0=b a$.

REMARK. We originally gave a spectral argument in [14] for $(2) \Rightarrow(3)$. We are indebted to $H$. Leptin for the neater version given below which is free of spectral considerations. We subsequently observed that Leptin's version of $(2) \Rightarrow(3)$ validates Lemma 2 for positive elements in the regular ring of a finite $A W^{*}$-algebra. Berberian has recently pointed out (in a letter to the author) that there are positive s.-a. elements in the regular ring with empty spectrum, so Leptin's version is significantly stronger.

Proof. (1) $\Rightarrow(2):(a+b)^{2}=|a-b|^{2}=(a-b)^{2}$, so $a b+b a=0$.

(3) $\Rightarrow(1):|a-b|=\left((a-b)^{2}\right)^{1 / 2}=\left(a^{2}+b^{2}\right)^{1 / 2}=a+b$, by uniqueness of the positive square root, so $a \wedge b=0$.

(2) $\Rightarrow(3): a^{2} b^{2}=b^{2} a^{2}$. But a positive operator has the same commutant as its square root, so $a b^{2}=b^{2} a$ and $a b=b a$. Finally, $a b=0=b a$. I

LEMma 3. Let $a, b, x, y$ be s.- $a$. operators with $0 \leqq x \leqq a, 0 \leqq y \leqq b$ and and $a b=0$. Then $x y=0$.

Proof. $0 \leqq a^{1 / 2} y a^{1 / 2} \leqq a^{1 / 2} b a^{1 / 2}=0$ so $a y=0$. But $0 \leqq y^{1 / 2} x y^{1 / 2} \leqq y^{1 / 2} a y^{1 / 2}=0$ and hence $x y=0$. I

Corollary 1. For two s.-a. operators $a$ and $b$, the following are equivalent:

(1) $|a|$ and $|b|$ are disjoint:

(2) $a b=0$;

(3) Any two of the operators $a^{+}, a^{-}, b^{+}, b^{-}$have zero product.

Proof. The equivalence of (1) and (3) is immediate from Lemmas 2 and 3 and the inequality $0 \leqq a^{+}, a^{-} \leqq|a|$. That (3) follows from (2) can easily be seen, for example, from a functional representation. I

LEMMA 4. Two s.-a. operators $a$ and $b$ commute if and only if each commutes with $a \bigwedge b$. 
Proof. Let $g=a \wedge b, u=a-g$ and $v=b-g$. Then $u=(a-b)^{+}$and $v=(a-b)^{-}($Lemma $1,(7))$, so $u \wedge v=0$ and by Lemma 2, $u v=0=v u$. Thus $(a-g)(b-g)=a b-a g-g b+g^{2}=0$ and $(b-g)(a-g)=b a-b g$ $-g a+g^{2}=0$. If $a g=g a$ and $b g=g b$ we have $a b=b a$. The converse is clear.I

Lemma 5. Let a be s.-a., e a projection with $0 \leqq a \leqq e$. Then $e a=a=a e$ (cf. [7]).

Proof. $a(1-e)=0$ by Lemma 3 . I

Proposition 1. For two projections $e$ and $f, e \wedge f \geqq 0$ if and only if $e$ and $f$ commute, in which case $e \wedge f=e \cap f$, the projection lattice GLB. Further, if ef $\neq f e$, then $e \wedge f$ is not comparable with zero.

Proof. Evidently, $e \wedge f \geqq 0$ if $e$ and $f$ commute. Conversely, for $g=e \wedge f$, we have $0 \leqq g \leqq e, f$. Hence $e g=g=g e$ and $f g=g=g f$ by Lemma 5 . By Lemma 4, ef $=f e=e \cap f$. But $|e-f|^{2}=(e-f)^{2}=(e-f)^{4}$, so $|e-f|$ $=(e-f)^{2}$ and $e \wedge f=e f=e \cap f$.

If $e f \neq f e$, then $e \wedge f \neq 0$ by the above. Suppose then that $e \wedge f \leqq 0$ and let us aim for a contradiction. Now $0 \leqq-(e \wedge f)=(-e) \bigvee(-f)$ so $e \leqq 0$ $\bigvee(e-f)$ and $f \leqq(f-e) \bigvee 0$, employing the translation invariance of $\bigvee$ (Lemma 1, (5)). But $(e-f)^{+}=(e-f) \bigvee 0$ and $(e-f)^{-}=(f-e) \bigvee 0$ so by Lemma 3 we get $e f=0$, contradicting ef $\neq f e$. Thus $e \wedge f \mid \neq 0$ as asserted. I

Corollary 2. For two s.-a. operators $a, b \geqq 0, a \wedge b \leqq 0$ implies that $a$ and $b$ are disjoint, so that $a b=0$.

Proof. The last part of the proof of Proposition 1, which did not use the fact that $e$ and $f$ are projections, applies equally to $a$ and $b$.

REMARks. For s.-a. operators $a$ and $b$ we have $a \wedge b \geqq 0$ if $a, b \geqq 0$ and $a$ and $b$ commute. But the rest of Proposition 1 may fail if $a$ and $b$ are not projections. For example, take

$$
a=\left(\begin{array}{ll}
1 & 0 \\
0 & 2
\end{array}\right) \text { and } b=\left(\begin{array}{ll}
1 & 1 \\
1 & 3
\end{array}\right) .
$$

Then $a, b \geqq 0, a \ddagger b, b \$ a$ and $a b \neq b a$. But

$$
a b+b a=\left(\begin{array}{cc}
2 & 3 \\
3 & 12
\end{array}\right) \geqq 0,
$$

so $(a-b)^{2} \leqq(a+b)^{2}$ and $|a-b| \leqq a+b$ (by monotonicity of the square root on positive operators). Thus $a \wedge b \geqq 0$.

From this last computation we obtain

COROLlary 3. Two projections commute if and only if their Jordan product is positive. For two s.-a. operators $a, b \geqq 0$, positivity of the Jordan product implies $a \wedge b \geqq 0$. 
Two s.-a. operators $a$ and $b$ will be called disjunctive if $|a-b| \wedge(a \wedge b)=0$. A disjunctive set of s.-a. operators is one in which every pair is disjunctive.

Proposition 2. Every disjunctive set of s.-a. operators is a commutative subset of the positive cone $S^{+}$of the space $S$ of all s.-a. operators. Each disjunctive set is contained in a maximal one and any maximal disjunctive set is a Boolean ring under the operations $\bigvee$ and $\Lambda$. A s.-a.operator $a$ is a projection if and only if 1 and $a$ are disjunctive. A disjunctive set containing the identity operator 1 consists entirely of projections.

Proof. If $|a-b| \wedge(a \wedge b)=0$, set $x=a-b$ and $g=a \wedge b$. By Lemma 1, (9) we have $0 \leqq g \leqq a, b$ since $|x| \wedge g \leqq g$. By Lemmas 2 and 3 we have $x^{+} g=0=x^{-} g$ and by Corollary $1, x g=0=g x$. Hence $a g=b g$ and $g a=g b$. But $a=x^{+}+g$ and $b=x^{-}+g$ by Lemma 1 , (7) so $a g=g^{2}=g b$. By Lemma $4, a b=b a$.

The second statement is an easy consequence of Zorn's Lemma. Now it is a classical fact that a maximal commutative set of s.-a. operators is a weakly closed algebra which forms a vector lattice under the operations $\vee$ and $\Lambda$. Thus any disjunctive set is contained in such an algebra, and the third statement can then be read out of [16].

Now $a b=g^{2}$ if $a$ and $b$ are disjunctive by the above computations. If 1 and $a$ are disjunctive we have $a=1 \cdot a=(1 \wedge a)^{2}$. But then $a^{1 / 2}=1 \wedge a \leqq 1$ and $0 \leqq a=a^{1 / 4} a^{1 / 2} a^{1 / 4} \leqq a^{1 / 2} \leqq 1$. Thus $1 \wedge a=a$ and $a^{2}=a$. Conversely, if $a^{2}=a$, we have $|1-a| \wedge(1 \wedge a)=(1-a) \wedge a=0$. I

3. Order properties. Let $V$ be a linear subspace of the space $S$ of all s.-a. operators and consider the following properties:

(A) If $a \in V$, then $|a| \in V$.

(L) The set $V^{+}$of positive operators in $V$ lattice orders $V$.

(T) $|a+b| \leqq|a|+|b|$, for all $a, b \in V$.

(P) $a \wedge b \geqq 0$ for $a, b \in V^{+}$.

(R) For $0 \leqq z \leqq a+b$ with $a, b, z \in V^{+}$, there exist $u, v \in V^{+}$with $z=u+v$, $0 \leqq u \leqq a$ and $0 \leqq v \leqq b$ (the Riesz Decomposition Property).

(SQ) $a^{2} \leqq b^{2}$ for $a, b \in V$ with $-b \leqq a \leqq b$.

(J) $a \circ b \geqq 0$ for $a, b \in V^{+}$(here $a \circ b=\frac{1}{2}(a b+b a)$ is the Jordan product).

(O) $a^{2} \leqq b^{2}$ for $a, b \in V$ with $0 \leqq a \leqq b$ (Ogasawara's condition).

$\left(\mathrm{J}^{+}\right) a \circ b \geqq 0$ for $a, b \in V$ with $0 \leqq a \leqq b$.

Lemma 6. If the operators in $V$ commute, then properties $(\mathrm{T}),(\mathrm{P}),(\mathrm{SQ}),(\mathrm{J})$, (O) and $\left(\mathrm{J}^{+}\right)$hold. Further $(\mathrm{O}) \Leftrightarrow\left(\mathrm{J}^{+}\right)$and $(\mathrm{L}) \Rightarrow(\mathrm{R})$. In the presence of $(\mathrm{A})$ we have $(\mathrm{J}) \Leftrightarrow(\mathrm{SQ}) \Leftrightarrow[(\mathrm{O})$ and $(\mathrm{P})]$.

Remark. In Proposition 6 below we show (T) $\Leftrightarrow$ (P) if (A) holds.

Proof. The first statement is a well-known consequence of the functional 
representation given by the Spectral Theorem.

$\left(\mathrm{J}^{+}\right) \Rightarrow(0)$ : Given $0 \leqq a \leqq b$. Then $0 \leqq b-a \leqq b+a$ and $b^{2}-a^{2}$ $=(b+a) \circ(b-a) \geqq 0$.

$(0) \Rightarrow\left(\mathrm{J}^{+}\right)$: For $0 \leqq a \leqq b$ we again have $0 \leqq b-a \leqq b+a$ so $(b-a)^{2}$ $\leqq(b+a)^{2}$. But then $a \circ b=\frac{1}{4}\left[(b+a)^{2}-(b-a)^{2}\right] \geqq 0$.

$(\mathrm{L}) \Rightarrow(\mathrm{R})$ is classical; one takes $u=\inf (z, a)$ and $v=z-u$.

Assume now that (A) holds in $V$.

$(\mathrm{J}) \Rightarrow(\mathrm{SQ})$ : For $-b \leqq a \leqq b$ we have $b+a, b-a \geqq 0$ and $b^{2}-a^{2}$ $=(b+a) \circ(b-a) \geqq 0$.

$(\mathrm{SQ}) \Rightarrow(\mathrm{P})$ : For $a, b \geqq 0,-(a+b) \leqq a-b \leqq a+b$, so $(a-b)^{2} \leqq(a+b)^{2}$.

Taking square roots preserves the order and $|a-b| \leqq a+b$. Thus $a \wedge b \geqq 0$.

$(\mathrm{SQ}) \Rightarrow(\mathrm{J})$ : Let $a, b \geqq 0$. Then $|a-b| \leqq a+b$, since (SQ) $\Rightarrow(\mathrm{P})$. But $(\mathrm{SQ}) \Rightarrow(0)$ trivially, so $(a-b)^{2}=|a-b|^{2} \leqq(a+b)^{2}$ and hence $a \circ b \geqq 0$.

$[(0)$ and $(\mathrm{P})] \Rightarrow(\mathrm{SQ}):$ Given $-b \leqq a \leqq b$. Then $b+a, b-a \geqq 0$ so $(b+a)$ $\Lambda(b-a) \geqq 0$ by $(\mathrm{P})$ and $|a| \leqq b$. By $(0), a^{2}=|a|^{2} \leqq b^{2}$. I

Definition. A linear space $V$ of s.-a. operators is called a special vector lattice if (A) and (L) hold in $V$ and if, in addition, $|a|=\sup (a,-a)$, where $|a|=\left(a^{2}\right)^{1 / 2}$ and "sup" denotes the least upper bound in the lattice order induced on $V$ by $V^{+}$.

If $V$ merely satisfies (L) we shall call $V$ a vector lattice, dropping the modifier "special."

A commutative linear space $V$ satisfying (A) is manifestly a special vector lattice. This is a minor variation of the classical fact mentioned already in the proof of Proposition 2. However, there are commutative vector lattices which are not special. Moreover there are noncommutative vector lattices, these being necessarily nonspecial, as we shall see presently. For examples of the above we refer the reader to [15].

It will be important for us to know when a linear space $V$ is a special vector lattice. We develop here a number of useful criteria.

Lemma 7. Suppose $a, b \geqq 0$ have a greatest lower bound $g$ relative to some linear space $V$ of s.-a. operators containing them, and that $a b=0$. Then $g=0$.

Proof. $0 \leqq g \leqq a, b$ so $g^{2}=0$ by Lemma 3 , and hence $g=0$.

LEMMA 8. Let $a, b \geqq 0$ have a greatest lower bound $g$ relative to a linear space $V$ of s.-a. operators, which contains $|a-b|$ as well as $a$ and $b$. Then $g=a \wedge b$ and hence, in particular, $a \wedge b \geqq 0$.

Proof. First we observe that existence of $g=\inf (a, b)$ implies existence of $\inf (a+c, b+c)$ for any $c \in V$. We have, moreover, $\inf (a+c, b+c)$ $=\inf (a, b)+c$. Thus $\inf (a-a \wedge b, b-a \wedge b)=g-a \wedge b$. But $(a-a \wedge b)$ $\wedge(b-a \wedge b)=0$ so $(a-a \wedge b)(b-a \wedge b)=0$ (Lemma 2). By Lemma 7 , we have $a \wedge b=g \geqq 0$. I 
The next proposition points out a certain superfluity in the definition of "special vector lattice."

Proposition 3. Let $V$ be a linear space of s.-a. operators which satisfies conditions (A) and (L). Then $V$ is a special vector lattice.

Proof. Given $a, b \in V$, let $g=\inf (a, b)$. By Lemma $8, g=a \wedge b$, and this is enough to insure that $V$ is special.

The following fact is classical and is easily verified.

Lemma 9. In any partially ordered real linear space $V$, property $(\mathrm{R})$ is equivalent to the statement:

For $x_{i} \leqq y_{k}$ with $x_{i}, y_{k} \in V(i, k=1,2)$, there is a $z \in V$ with $x_{i} \leqq z \leqq y_{k}$.

Lemma 10. Let $V$ be a linear space of s.-a. operators which has property (R). If $a, b \geqq 0$ in $V$ are such that $a b=0$, then $a$ and $b$ have greatest lower bound zero in $V$.

Proof. Take $x \in V$ with $x \leqq a, b$. Then by Lemma 9, there is a $z \in V$ with $0, x \leqq z \leqq a, b$. By Lemma 3 we have $z^{2}=0$, and $z=0$. But then $x \leqq 0$ and hence $\inf (a, b)=0.1$

Proposition 4. If $V$ is a linear space of s.-a. operators which satisfies conditions $(\mathrm{A})$ and $(\mathrm{R})$, then $V$ is a special vector lattice.

Proof. This is immediate from Lemma 10.

Lemma 11. Let $V$ be a linear space of s.-a. operators in which $(0)$ holds. Then for $a, b \geqq 0$ in $V$ with $a b=0, a$ and $b$ have greatest lower bound zero in $V$.

Proof. We have remarked already in Corollary 3 that for $a, b \geqq 0, a \circ b \geqq 0$ implies $a \wedge b \geqq 0$. Also $\left(\mathrm{J}^{+}\right)$is equivalent to (0) by Lemma 6 .

- Given $x \in V$ with $x \leqq a, b$. Then $0 \leqq a \leqq a+b-x$ and $0 \leqq b \leqq a+b-x$, since $a-x, b-x \geqq 0$. Now by $\left(\mathrm{J}^{+}\right), a \circ(a-x)=a \circ(a+b-x) \geqq 0$ and $b \circ(b-x)=b \circ(a+b-x) \geqq 0$ so by the remark at the beginning of the proof we have $a \wedge(a-x) \geqq 0$ and $b \wedge(b-x) \geqq 0$. But $a-x^{+}=a+$ $(0 \wedge(-x))=a \wedge(a-x) \geqq 0$ and similarly $b-x^{+} \geqq 0$. Thus $0 \leqq \dot{x}^{+} \leqq a, b$. By Lemma $3, x^{+}=0$ so $x=-x^{-} \leqq 0$ and we have $\inf (a, b)=0$.

Proposition 5. If $V$ is a linear space of s.-a. operators which has properties (A) and (0), then $V$ is a special vector lattice.

Proof. This follows at once from Lemma 11. I

Proposition 6. For a linear space $V$ of s.-a. operators, the following are equivalent:

(1) $V$ is a special vector lattice.

(2) $V$ is a semigroup under $\wedge$ (or $\bigvee$ ).

(3) V satisfies conditions (A) and (T). 
(4) V satisfies conditions (A) and (P).

(5) V satisfies condition (A) and $|a| \leqq b$, whenever $-b \leqq a \leqq b$ with $a, b \in V$.

Proof. The implications $(1) \Rightarrow(n)$ for $n=2, \cdots, 5$ are clear.

$(2) \Rightarrow(1)$ : It suffices to show that $a \wedge b$ is the greatest lower bound of $a$ and $b$ in $V$. But if $x \in V$ with $x \leqq a, b$ we have by Lemma $1,(1), a \wedge x=x$ $=b \wedge x$. Now $x \wedge(a \wedge b)=(x \wedge a) \wedge b=x \wedge b=x$, so $x \leqq a \wedge b$ as required. The proof for $\bigvee$ is dual.

In each of the remaining cases, assume $0, a \leqq b$. We shall show $a^{+} \leqq b$.

(3) $\Rightarrow(1)$ : We have $b, b-a \geqq 0$ and $a=b-(b-a)$ so $|a| \leqq|b|+|b-a|$ $=2 b-a$. Thus $a^{+} \leqq b$.

(4) $\Rightarrow$ (1): Again $b, b-a \geqq 0$ and $b-a^{+}=b \wedge(b-a) \geqq 0$.

(5) $\Rightarrow(1):-2 b+a \leqq a \leqq 2 b-a$, so $|a| \leqq 2 b-a$ and $a^{+} \leqq b$. I

4. Some facts about pre- $(M)$-spaces. The main purpose of this section is to collect a number of results (mostly familiar) needed in the sequel.

Lemma 12. Any special vector lattice is a pre-( $M)$-space in the operator norm.

Proof. Recall first that a pre- $(M)$-space is a vector lattice with a norm satisfying: (i) $\|a\| \leqq\|b\|$ for $|a| \leqq|b|$; and (ii) $\|a \bigvee b\|=\|a\| \bigvee\|b\|$ for $a$, $b \geqq 0$. By Lemma $1,(10)$ the first condition holds throughout $S$. According to [3], it is enough to show that (ii) holds with $a \wedge b=0$. But in this case $a$ and $b$ commute and by the Spectral Theorem we may choose a functional representation of the smallest uniformly closed algebra of s.-a. operators containing $1, a$ and $b$. This algebra is represented as a real $C(X)$ (with $X$ a compact subset of the plane) and the representation preserves absolute value, products, the linear structure and the norm. This information, together with the fact that $C(X)$ is an $(M)$-space, concludes the proof. I

Now consider an arbitrary vector lattice $V$ with a norm satisfying $\|a\|$ $\leqq\|b\|$ whenever $|a| \leqq|b|$ in $V$. A state of $V$ is a positive linear functional of bound $\leqq 1$. From the Alaoglu Theorem, we see that the set of all states is a $w^{*}$-compact convex subset of the norm unit ball of $V^{*}$. Denote by $\mathscr{P}$ the set of extreme ("pure") states and let $X$ be the $w^{*}$-closure of $\mathscr{P}$ with zero state deleted (it is always pure). Now let $\mathscr{L}$ be the set of "lattice states," i.e., those states which preserve absolute value. It is easy to see that $\mathscr{L}$ is $w^{*}$-compact (it may consist of the zero state alone). In order that $\mathscr{P} \subset \mathscr{L}$ it is necessary and sufficient that property (ii) (see the proof of Lemma 12 above) hold, i.e., that $V$ be a pre- $(M)$-space. If $\mathscr{P} \subset \mathscr{L}$ then $X \subset \mathscr{L}$. We shall refer to $X$ as the pure state space of $V$. Representing $a \in V$ by the function $a^{\prime}(x)=x(a)$, where $x \in X$ we obtain a linear lattice preserving isometry of $V$ with a linear sublattice of $C(X)$. Then $X$ is locally compact, all functions in $V$ vanish at infinity (the zero state), $V$ separates points of $X$ and $\infty$ is the only point where all functions in $V$ vanish. This is the pure state representation of $V$. We summarize this in 
ThEOREM 1 (KAKUTANI). The following are equivalent:

(1) $V$ is a pre- $(M)$-space.

(2) $V$ is a linear sublattice of $C(X), X$ locally compact (the pure state space of $V)$, which separates the points of $X$. Each function in $V$ vanishes at $\infty$, and $\infty$ is the only point where all functions in $V$ vanish.

Kakutani proved more (see [4, p. 103, Theorem 4], for an account and references), but this suffices for our purposes.

Proposition 7. Let $V$ be a pre-(M)-space, $X$ its pure state space and $P$ the positive part of the norm unit ball in $V$. Define a function $e$ on the pure state space by the formula

$$
e(x)=\sup \{f(x): f \in P\} .
$$

Then $f \in P$ is an extreme point of $P$ if and only if $f$ satisfies:

(E) For each $x \in X, f(x)>0$ implies $f(x)=e(x)$.

REMark. Although $e$ is finite-valued and lower semi-continuous, it need not be in $V$ or even in $C(X)$.

Proof. If $f$ has property (E), then let $f=\frac{1}{2}(g+h)$ with $g, h \in P$. If $f(x)=0$ then $g(x)=0=h(x)$. If $f(x)>0$ then $f(x)=e(x)$ and $g(x), h(x) \leqq e(x)$. Suppose one of $g(x), h(x)$ is $<e(x)$. Then $e(x)=\frac{1}{2} g(x)+\frac{1}{2} h(x)<\frac{1}{2} e(x)$ $+\frac{1}{2} e(x)=e(x)$, a contradiction. Hence $g(x)=e(x)=h(x)$, so $g=f=h$ and $f$ is extreme.

Conversely, suppose (E) fails for $0 \neq f \in P$ ( 0 is always extreme). By assumption, there is an $a \in X$ with $0<f(a)<e(a)$. Moreover, we can find a function $k \in P$ with $f(a)<k(a)$, for otherwise we would have $f(a)=k(a)$ $=e(a)$. Now set $g=f \bigvee k$. Then $f<g \in P$. Take $h=g \wedge 2 f$. Then $h \in P$ since $0 \leqq h \leqq g \leqq 1$; moreover, $0 \leqq h \leqq 2 f$. Also $2 f \leqq 1+f \leqq 1+h$ since $f \leqq h$ (because $f \leqq g$ and $f \leqq 2 f$ ). Thus $0 \leqq 2 f-h \leqq 1$ and hence $h, 2 f-h$ $\in P$. But $f=\frac{1}{2} h+\frac{1}{2}(2 f-h)$. Evaluating at the point $a$, we see that $f \neq h$, so $f$ is not extreme. $I$

COROLlary 4. Let $V$ be a special vector lattice of s.-a. operators. The set $E$ of extreme points of $P$ forms a Boolean ring under $\bigvee$ and $\Lambda$, so any two extreme points of $P$ commute.

Proof. In the pure state representation of $V$, Proposition 7 describes the extreme points of $P$ as those functions $f \in P$ which take either the value 0 or $e(x)$ at a point $x \in X$ (and only these values). For $f, g \in E,(f \bigvee g)(x)$ $=e(x)$ if $f(x)>0$ or $g(x)>0$ and $(f \bigvee g)(x)=0$ otherwise. Also $(f-(f \wedge g))$ $\cdot(x)=e(x)$ if $f(x)>0$ and $g(x)=0$; otherwise we have $(f-(f \wedge g))(x)=0$. Thus $f \bigvee g, f-(f \wedge g) \in E$. From [16] (proof of Lemma 2), $E$ is a Boolean ring under $\bigvee$ and $\Lambda$ with $f-(f \wedge g)$ as the relative complement operation. Being disjunctive, $E$ is commutative by Proposition 2 . 
5. The bounded strong operator topology. In the space $S$ of all s.-a. operators (or in the ring of all bounded operators) we define a topology called the bounded strong operator topology as follows. A set $E \subset S$ is closed if and only if $E \cap B$ is strongly closed (i.e., closed in the strong operator topology) in $B$, for each norm-bounded set $B \subset S$. The bounded strong closure of $E$ will be denoted by $b s(E)$.

The bounded weak operator topology, et al., can be defined similarly, but we shall have no occasion to consider these notions here. Concerning such topologies, we refer the reader to [4, Chapter II, \$5, especially pp. 41-43]. A few relevant facts are listed below without proof; the proofs are isomorphic to ones given in [4].

LEMMA 13. $\operatorname{bs}(E)$ is the set of all limits of strongly convergent norm-bounded nets from $E$. The bounded strong, strong and weak closures of a norm-bounded convex set coincide.

The weak and strong closures of a convex set of operators coincide by [6]. If $\mathrm{s}(E)$ denotes the strong closure of $E$, then in general, we have $\mathrm{s}(E) \supset \mathrm{bs}(E)$. One might suspect that $\mathrm{s}(K)=\mathrm{bs}(K)$ for any convex set $K \subset S$, but this is not the case. One need only take $K$ to be the null space of an ultrastrongly continuous, but strongly discontinuous, linear functional on $S$. Such functionals exist by Théorème 1 (p. 40) of [5].

Lemma 14. $A$ set $E$ is bs-closed (open) if and only if $E \cap U$ is relatively strongly closed (open) in $U$, for every norm ball $U$ about zero in $S$.

For the proof, see Lemma 2 (p. 42) of [4].

CoROLlary 5. The bounded strong operator topology is locally convex. If $\mathrm{u}$, bs and s denote, respectively, the uniform, bounded strong and strong operator topologies, then $\mathrm{s} \subset$ bs $\subset \mathrm{u}$.

The map $a \rightarrow a^{2}$ from $S$ to itself is known to be discontinuous in both the weak and strong operator topologies (see [5, Chapter I, \$3]; this result seems to be due to $\mathrm{E}$. Michael). This is our motivation for considering the bounded strong operator topology.

Proposition 8. The operations $a \rightarrow a^{2},(a, b) \rightarrow a b+b a$ and $a \rightarrow|a|$ are continuous in the bounded strong operator topology. If $f$ is any continuous real function (not necessarily bounded) of a real variable, the map $a \rightarrow f(a)$ from $S$ to itself is continuous in the bounded strong operator topology.

Proof. This follows from a result of Kaplansky [9, Corollary to Theorem 2]. Officially, Kaplansky is referring to the strong continuity of a bounded real continuous function of a s.-a. operator over all of $S$. However, the same effect is achieved if we restrict a not necessarily bounded real continuous 
function (such as the absolute value) of a s.-a. operator to norm-bounded sets (we are indebted to J. Dixmier for calling our attention to this device).

Proposition 9. The bounded strong closure of a special vector lattice is again a special vector lattice.

Proof. This is immediate from the joint continuity of the lattice operations in the bounded strong operator topology and this, in turn, follows from the bounded strong continuity of the absolute value (Proposition 8). I

6. Special vector lattices. We are now in a position to obtain our main result and to draw from it a number of nontrivial conclusions.

THEOREM 2. Every special vector lattice of s.-a. operators is commutative.

Proof. Let $V$ be a special vector lattice and let $L=\operatorname{bs}(V)$ be its bounded strong closure. Then $L$ is an $(M)$-space (Proposition 9, Lemma 12 and Corollary 5). It will turn out that $L$ also has an order unit (see the proof of Corollary 6) and is therefore representable as a full $C(X), X=$ the compact pure state space of $L$, but this, and the fact that $L$ is uniformly closed, is not needed for the commutativity result. Let $P$ be the positive part of the norm unit ball in $V$. Then bs $(P)=\mathrm{s}(P)=\mathrm{w}(P)$ (the weak closure of $P$ ) by Lemma 13 , since $P$ is obviously convex. Let $Q=\mathrm{w}(P)$. Then $Q \subset L$ and $Q$ (manifestly convex) is compact in the weak operator topology $[5, \mathrm{p}$. 34]. The Krein-Milman Theorem now tells us that $Q$ is the weakly closed convex hull of its set $E$ of extreme points. By Corollary 4 , the set $E$ is a commutative Boolean ring of s.-a. operators, and hence $E$ is contained in a maximal commutative set $A$ of s.-a. operators. But $A$ is evidently a (weakly closed) maximal abelian algebra of s.-a. operators and the Krein-Milman result shows that $L \subset A$. Thus $L$ is commutative. I

Corollary 6. Let $V$ be any special vector lattice, $L=\mathrm{bs}(V)$ its bounded strong closure, $P$ the positive part of the norm unit ball of $V, Q=\operatorname{bs}(P)$ its bounded strong closure. Then $Q$ contains a largest (in the ordering of s.-a. operators) operator $e$, and $e$ is an order unit for $L$. The special vector lattice $L$ is a boundedly complete $(M)$-space and is isometric and linear lattice isomorphic to a $C(X)$, with $X$ compact and extremally disconnected (but not necessarily measure bearing).

Proof. The set $Q$ is directed, in fact, lattice ordered, by $\leqq$ and bounded above in $S$ by the identity operator $1 \in S$. Thus $Q$ has an LUB $e \in S$ which is both a weak and strong limit point of $Q$ (regarded as a net); see [5, Appendice II]. Thus $e \in Q$ and $e$ is evidently an order unit for $L$ since the absolute value of any $a \in L$ is dominated by $\|a\| e$. Essentially the same arguments show that any set in $L$ which is bounded above in $L$ has an LUB in $L$ so that $L$ is boundedly complete. A well-known result of $M$. H. Stone [12] now 
implies that the (normalized) pure state space $X$ of $L$ is extremally disconnected. Note, however, that $X$ need not be "hyperstonian" (in Dixmier's terminology). That $L$ exhausts $C(X)$ can be read out of $[13$, Corollary 3 , p. 174]. I

Corollary 7. Let $V$ be a special vector lattice, $L=\operatorname{bs}(V)$ as in Corollary 6 , with e the order unit described there. Then $L$ is an algebra of s.-a. operators if and only if $e$ is a projection.

Proof. If $e$ is a projection, it can, by reduction of the underlying Hilbert space, be assumed to be the identity operator. By Proposition 2, the set $E$ of extreme points of $Q$ is a Boolean algebra of projections and this obviously makes $L$ an algebra, since the linear hull of $E$ is a bounded strong dense algebra in $L$.

Conversely, if $L$ is an algebra, then $e^{2} \in Q$, since $e^{2} \leqq e \leqq 1$ and $e^{2} \in L$. But $e$ is an extreme point of $Q$ by Proposition 7. Now $(1-e)^{2}=1-2 e+e^{2}$ $\geqq 0$ so $0 \leqq 2 e-e^{2} \leqq 1$ and hence $2 e-e^{2} \in Q$. Since $e=\frac{1}{2} e^{2}+\frac{1}{2}\left(2 e-e^{2}\right)$, we must have $e^{2}=e$. I

We now employ the criteria developed in $\$ 3$ to obtain several applications of Theorem 2.

Corollary 8 (Sherman's Theorem [11]). If the s.-a. operators in a $C^{*}$ algebra $A$ are lattice ordered by $A^{+}$, then $A$ is commutative.

Proof. This is immediate from Proposition 3 and Theorem 2. I

Corollary 9 (Theorem of Fukamiya-Misonou-Takeda [7]). If $A$ is a $C^{*}$-algebra whose set of s.-a. operators has property $(\mathrm{R})$ of $\$ 3$, then $A$ is commutative.

Proof. This follows at once from Proposition 4 and Theorem 2. I

Corollary 10 (Ogasawara's Theorem [10]). If $A$ is a $C^{*}$-algebra whose set of s.-a. operators has property $(0)$, then $A$ is commulative.

Proof. We apply Proposition 5 in conjunction with Theorem 2. I

CoRollary 11. Let $V$ be a linear space of s.-a. operators satisfying condition (A) of $\$ 3$. Then the statements: " $V$ has property $(\mathrm{X})$," where $(\mathrm{X})$ is any one of the properties $(\mathrm{L}),(\mathrm{T}),(\mathrm{P}),(\mathrm{R}),(\mathrm{SQ}),(\mathrm{J}),(\mathrm{O})$ or $\left(\mathrm{J}^{+}\right)$, are equivalent and each statement is, moreover, equivalent to the commutativity of $V$.

Proof. This is a reinterpretation of the facts uncovered in Lemma 6, Proposition 4 and Theorem 2. I

Corollary 12. Let $S$ and $T$ be the spaces of all s.-a. operators on the Hilbert spaces $H$ and $K$, respectively. Let $A$ be any linear subspace of $S$ which satisfies condition (A) of $\$ 3$ and suppose that $\phi$ is a linear map of $A$ into $T$ which satis- 
fies $\phi(|a|)=|\phi(a)|$, for all $a \in A$. Then $\phi$ preserves commutativity (but $\phi$ may destroy squares).

Proof. Suppose $a, b \in A$ commute. Let $V$ be any special vector lattice in $S$ containing $a$ and $b$ (e.g., the smallest uniformly closed algebra of s.-a. operators containing $a$ and $b$ ). Then $W=V \cap A$ is also a special vector lattice since $A$ is closed under $a \rightarrow|a|$. The image of $W$ under $\phi$ is also a special vector lattice containing $\phi(a)$ and $\phi(b)$, so these operators commute. I

7. Connections with Jordan algebras. Linear subspaces of the space $S$ of all s.-a. operators which are closed under the operation $a \rightarrow|a|$ (property (A)) bear, in some respects, a resemblance to Jordan algebras of s.-a. operators (linear subspaces of $S$ closed under $a \rightarrow a^{2}$ ). The extent of the similarity seems to depend on the "proximity" of the subspace to the identity operator. We make this precise below, but we need to dispense with some technicalities first.

If $X$ is a set and $A$ is a set of real-valued functions on $X$, we say that $A$ has the two-point property if for any reals $\alpha$ and $\beta$ and any two distinct points $x$ and $y$ in $X$, there is a function $f \in A$ with $f(x)=\alpha$ and $f(y)=\beta$.

Lemma 15. Let $X$ be a compact subset of $[0,1]$ and let $A \subset C[0,1]$ be a linear subspace containing the "diagonal function" $f(t)=t$ and its integral truncations $1 \wedge(n f)(n=2,3, \cdots)$. Then $A$ has the two-point property.

Proof. Given $0<x<y$ in $X$ and reals $\alpha, \beta$. Take $n$ so that $n^{-1}<x$ and choose reals $\lambda$ and $\mu$ so that the function $h=\lambda f+\mu[(n f) \wedge 1]$ takes the value $\alpha$ at $x$ and $\beta$ at $y$. This can be done, since $h(x)=\lambda x+\mu$ and $h(y)=\lambda y+\mu$; we simply solve the equations

for $\lambda$ and $\mu . \mid$

$$
\begin{aligned}
& \lambda x+\mu=\alpha, \\
& \lambda y+\mu=\beta
\end{aligned}
$$

Lemma 15 is a special variant of a rather general related fact: Let $X$ be any set and let $A$ be a vector lattice of real-valued functions on $X$ (pointwise linear and lattice operations). Assume further that (1) $A$ separates the points of $X$; (2) there is no point of $X$ at which every function in $A$ vanishes; (3) $A$ contains $1 \wedge f$, whenever it contains $f \geqq 0$ (by virtue of the relation $1 \wedge f=1$ $\wedge|f|-\left(1 \wedge f^{-}+f^{-}\right),(3)$ is equivalent to Stone's "measurability condition": $1 \wedge f \in A$ for all $f \in A$ ). Then $A$ has the two-point property.

The proof of this last fact is not difficult, but we omit the details.

LEMMA 16. In $C[0,1]$, the functions $h$ satisfying $h(0)=0$ and $x h(y) \neq y h(x)$ for all $0<x<y \leqq 1$ are precisely the strictly convex or strictly concave functions which vanish at zero.

The proof is elementary and is omitted. 
We need to cite a theorem of M. H. Stone on vector lattices of continuous functions on a locally compact space. Since Stone's formulation of this theorem in [13, Theorem 12] contains a flaw, we list some alternatives.

Stone's Theorem. Let $E$ be a locally compact Hausdorff space, $C_{\infty}(E)$ the algebra of all real continuous functions on $E$ which vanish at infinity. If $A$ is a uniformly closed linear sublattice of $C_{\infty}(E)$ then any one of the conditions below implies $A=C_{\infty}(E)$.

(1) $A$ has the two-point property.

(2) $A$ separates points of $E$, there is no point of $E$ at which every function in $A$ vanishes and $1 \wedge f \in A$, whenever $f \in A, f \geqq 0$.

(3) $A$ separates points of $E$, there is no point of $E$ at which every function in $A$ vanishes and $f^{2} \in A$ if $f \in A$.

Stone's assumptions in [13] are (i) $A$ separates points of $E$; and (ii) there is no point of $E$ where every function in $A$ vanishes. To see that these conditions alone do not suffice, take $E=(0,1]$ and let $A$ be the one-dimensional vector lattice of all real multiples of the "diagonal function" $f(t)=t$. Then $A$ satisfies (i) and (ii), but $A \neq C_{\infty}(E)$.

Having disposed of these preliminaries, we come to the main result of this section.

TheOREM 3. Let $V$ be a linear space of s.-a. operators which is closed under the operation $a \rightarrow|a|$ and let $A$ be its uniform closure. Then any one of the conditions below implies that $A$ is a Jordan algebra.

(1) $1 \in A$.

(2) $1 \wedge a \in A$, for each $a \in V^{+}$(Stone's "measurabilitycondition").

(3) For each $a \in V$ with $0 \leqq a \leqq 1$, there is a strictly convex or strictly concave continuous function $h$ on $[0,1]$ with $h(0)=0$ (depending on a) such that $h(a) \in A$.

Proof. For any s.-a. operator $a \in V$, let S(a) (the "spectral algebra" of $a$ ) be the uniform closure of the real polynomials in $a$ without constant term. Let $\operatorname{sp}(a)$ denote the spectrum of $a$. Then for $0 \leqq a \leqq 1, \mathrm{~S}(a)$ has a distinguished functional representation either as $(1) \mathrm{C}(\operatorname{sp}(a))$, the algebra of all real continuous functions if $0 \notin \mathrm{sp}(a)$; or as $(2) \mathrm{C}_{0}(\operatorname{sp}(a))$, the algebra of all real continuous functions vanishing at zero if $0 \in \operatorname{sp}(a)$. In any case, $a$ is represented by the "diagonal function" $a(t)=t$ on $\operatorname{sp}(a) \subset[0,1]$. Moreover, it is clear that the image $A(a)$ of $A \cap \mathrm{S}(a)$ in $\mathrm{C}(\operatorname{sp}(a))$ separates the points of $\operatorname{sp}(a)-\{0\}$; and there is no point of this set at which every function in $A(a)$ vanishes.

Regarding $A(a)=A \cap \mathrm{S}(a)$, our task is finished if we can show that $A(a)$ $=\mathrm{S}(a)$. For then $a^{2} \in A$ if $a \in V$ with $0 \leqq a \leqq 1$, and this last restriction is removed by normalizing and writing $a=a^{+}-a^{-}$. For $a \in A, a^{2} \in A$ then follows from the uniform density of $V$ in $A$. 
If $1 \in A, A(a)=\mathrm{S}(a)$ follows at once from the usual Stone-Weierstrass Theorem (Stone's lattice version).

In the other two cases $(1 \notin A)$ it is enough to show that $A(a)$ has the twopoint property on $\operatorname{sp}(a)-\{0\}$. If $(2)$ holds, this is a consequence of Lemma 15.

Finally, suppose (3) holds. Let $x, y \in \operatorname{sp}(a), 0<x<y$, and let reals $\alpha$ and $\beta$ be given. Then there is a function $h \in C[0,1]$ with $h(0)=0$ and $x h(y)$ $\neq y h(x)$. The system

$$
\begin{aligned}
& \lambda x+\mu h(x)=\alpha, \\
& \lambda y+\mu h(y)=\beta
\end{aligned}
$$

of linear equations has $x h(y)-y h(x) \neq 0$ as its determinant and can therefore be solved for $\lambda$ and $\mu$. The function $g(t)=\lambda a(t)+\mu h(a(t))$ has the desired two-point property and is in $A(a)$.।

Theorem 3 is, by contrast, a "noncommutative" result. We state its commutative counterpart separately as

COROLLARY 13. Let Vbe a special vector lattice and let $A$ be its uniform closure. Then any one of conditions (1), (2) or (3) of Theorem 3 implies that $A$ is an algebra of s.-a. operators.

8. Unbounded operators. Throughout this section, $A$ will denote either a finite von Neumann algebra or an $A W^{*}$-algebra of finite type and $M$ its regular ring in the sense of [1]. In the concrete case, $M$ is the ${ }^{*}$-algebra of measurable operators constructed over $A$ (see $[1$, p. 228]).

By Corollary 6.2 of [1], any positive element in $M$ has a unique positive square root in $M$. Thus for any s.-a. element $a \in M$ we may define $|a|$ $=\left(a^{2}\right)^{1 / 2}$ as before and note that our definition of "special vector lattice" is thus extended to linear subspaces $V$ of s.-a. elements in $M$ satisfying (for example) conditions (A) and (T) of $\S 3$.

In the $A W^{*}$-case, we shall impose one further restriction. We do not know whether this is necessary, but lacking more information about the regular ring, we shall content ourselves with this rather reasonable assumption. This is

(MCP) Every increasingly directed family of s.-a. operators in $A$ which is bounded above in $A$ has a least upper bound in $A$.

Berberian [2, Corollary to Theorem 6] has shown that $M$ also has the "monotone convergence property" (MCP) if $A$ does. The primary motive for assuming (MCP) is provided by the following recent result of Berberian (communicated in a letter to the author); the proof will appear elsewhere.

Proposition 10. If the $A W^{*}$-algebra $A$ (and hence also its regular ring $M$ ) satisfies $(M C P)$, then the square root operation preserves order in $\mathrm{M}^{+}$, i.e., for $a \leqq b$ with $a, b \in M^{+}$we have $a^{1 / 2} \leqq b^{1 / 2}$. 
From this, and the results of [1], we immediately conclude

Lemma 17. Lemmas 1 (1)-(9), 2, 3, 4, 6, 7, 8, 10, 11, Propositions 2, 3, 4, 5, 6 and Corollaries 1, 2 and 3 (last half) remain valid in the regular ring $M$ of a finite $A W^{*}$-algebra satisfying (MCP).

Proof. Very few additional remarks are needed. These are:

Lemмa 3. Use the remark preceding Definition 6.2 in [1].

Lemma 6. Use Proposition 10 and original proof.

Corollary 1. The proof of $(2) \Rightarrow(3)$ can be obtained directly as follows. If $a b=0(=b a)$, then $a b^{2}=b^{2} a=0$ and $a|b|=|b| a$ by Corollary 6.2 of [1]. Thus a commutes with $b^{+}$and $b^{-}$; also $a b^{+}=a b^{-}$. Now $\left(a b^{+}\right)^{2}=\left(a b^{+}\right)\left(a b^{-}\right)$ $=a^{2} b^{+} b^{-}=0$ (Lemma 2), so $a b^{+}=0$; similarly $a b^{-}=0$. Repeating the argument gives $a^{+} b^{+}=a^{+} b^{-}=a^{-} b^{+}=a^{-} b^{-}=0$.

Corollary 3 (LASt half). Proposition 10 is needed here again. I

If $V$ is a linear subspace of s.-a. elements in the regular ring $M$, which is closed under $a \rightarrow|a|$, then to show that $V$ is commutative, it is enough to show that any two positive elements in $V$ commute, by virtue of the Jordan decomposition $a=a^{+}-a^{-}$.

Now let $a \in M^{+}$and define the bounded transform $a_{0}$ of $a$ by the formula: $a_{0}=a(1+a)^{-1}$. By Lemma 1 of [2] we have $(1+a)^{-1} \geqq 0$, since $a \geqq 0$ and by the remarks after Definition 6.3 of [1], we have $a_{0} \geqq 0$. Also

$$
1-a_{0}=((1+a)-a)(1+a)^{-1}=(1+a)^{-1} \geqq 0,
$$

so $a_{0} \leqq 1$ and hence $a_{0}$ lies in the bounded algebra $A$ by Lemma 5.1 of [1]. Obviously, $(1+a)^{-1}, a(1+a)^{-1} \in\{a\}^{\prime \prime}$.

We shall define the "spectral family" ("spectral projections," etc.) of an element $a \in M^{+}$to be the (ordinary) spectral family (spectral projections, etc.) of the bounded transform $a_{0}$ of $a$. A number of other, perhaps more sophisticated, alternatives are available - we might employ the Cayley transform instead of the bounded transform, for example - but our notion is adequate and somewhat simpler.

LEMMA 18. For two elements $a, b \geqq 0$ in the regular ring, the following are equivalent:

(1) $a b=b a$;

(2) $a_{0} b=b a_{0}$;

(3) $a_{0} b_{0}=b_{0} a_{0}$;

(4) Each "spectral projection" of a commutes with each "spectral projection" of $b$.

(5) Each spectral projection of $a_{0}$ commutes with each spectral projection of $b_{0}$.

Proof. The implications $(1) \Rightarrow(n)$ for $n=2, \cdots, 5$ are clear and $(4) \Leftrightarrow(5)$ 
by definition. The Spectral Theorem yields (5) $\Rightarrow(3)$.

It is enough, therefore, to show that (2) implies (1). If $a_{0} b=b a_{0}$, then $(1+a)^{-1}(a b)=(b a)(1+a)^{-1}$, so that $a b+a b a=(a b)(1+a)=(1+a)(b a)$ $=b a+a b a$ and finally $a b=b a .1$

From these facts, we deduce a simple criterion for commutativity.

Theorem 4. Let $V$ be a special vector lattice of s.-a. elements in the regular ring $M$ and assume that $V$ has the property:

For each $a \in V^{+}, V$ contains the "spectral projections" of $a$.

Then $V$ is commutative.

Proof. Let $a, b \in V^{+}$. Then the spectral projections of $a_{0}$ and $b_{0}$ are in $V$. By Proposition 1 of $\S 2$, any two projections $e, f$ in a special vector lattice commute, because $e \wedge f \geqq 0$. Thus $a b=b a$ by Lemma 18 and $V$ is commutative. 1

REMARK. Theorem 4 is true without assuming the "monotone convergence property" (MCP) on $A$.

We have already noted that $a_{0} \in\{\mathrm{a}\}^{\prime \prime}$ for $a \in M^{+}$. The reverse is also true: $a \in\left\{a_{0}\right\}^{\prime \prime}$, so that, in fact, $\left\{a_{0}\right\}^{\prime \prime}=\{a\}^{\prime \prime}$. For if $x \in M$ and $x a_{0}=a_{0} x$, then $x$ can be assumed positive; this is because $\left\{a_{0}\right\}^{\prime}$ is a s.-a. subalgebra of $M$ closed under $x \rightarrow|x|=\left(x^{*} x\right)^{1 / 2}$ (see Corollary 6.2 of [1]). But then $x \in\{a\}^{\prime}$ by Lemma $18,(2) \Rightarrow(1)$.

These computations lead us to

TheOREM 5. Let $A$ be a finite $A W^{*}$-algebra having the "monotone convergence property" $(M C P), M$ its regular ring. Let $R$ be ${ }^{*}$-subalgebra of $M$ and suppose that $R=R^{\prime \prime}$ (commutants relative to $M$ ). Then any one of the properties: (L), (T), (P), (R), (SQ), (J), (O) or $\left(\mathrm{J}^{+}\right)$implies the commutativity of $R$.

Proof. The set $V$ of s.-a. elements of $R$ is closed under $a \rightarrow|a|$, since $|a|$ $\in\{a\}$ " by Corollary 6.2 of [1]. By Lemma 17, any of the properties in question imply that $V$ is a special vector lattice of s.-a. elements in $M$. For $a \in V^{+}$, the s.-a. part of $\{a\}^{\prime \prime}$ is contained in $V$, since $R=R^{\prime \prime}$. Since $\left\{a_{0}\right\}^{\prime \prime}$ $=\{a\}^{\prime \prime}$, the spectral projections of $a_{0}$ all lie in $V$ and Theorem 4 applies, yielding commutativity. I

Theorem 5 can also be formulated for Jordan algebras of s.-a. elements in the regular ring. One then deals with the s.-a. part of $\{a\}^{\prime \prime}$.

A final result in this area is contained in the next theorem. For this, assume that $A$ is a von Neumann algebra of type $\mathrm{II}_{1}$ having a faithful numerical trace. Thus $A$ may be either a type $\mathrm{II}_{1}$ algebra with countably decomposable center or a $\mathrm{II}_{1}$-factor. Let $L^{1}$ denote the space of summable operators in $M$ arising from the trace on $A$.

THEOREM 6. Every special vector lattice of s.-a. operators which is contained in $L^{1}$ is commutative and its trace norm closure is an abstract $(L)$-space (also commutative). 
Proof. Let $V$ be a special vector lattice with $V \subset L^{1}$ and let $L$ be the trace norm closure of $V$. Since $a \rightarrow|a|$ is clearly continuous in the trace norm topology, $L$ is also a special vector lattice. Evidently, then, $L$ is an abstract $(L)$-space in the sense of Kakutani (see $[4$, p. 100] for references), the norm being the trace norm $\|a\|_{1}=$ trace $(|a|)$. By Kakutani's Theorem (see [4, p. 107, Theorem 2]), abstract $(L)$-spaces are concrete. We therefore regard $L$ as concrete and consider the Boolean ring $E \subset L^{+}$consisting of Lebesgue classes of characteristic functions of measurable sets of finite measure. By Proposition 2 of $\S 2, E$ is commuting, being a disjunctive set. But the linear hull of $E$ is trace norm dense in $L$, so that $L$ is commutative.

9. Acknowledgement. Special thanks are due Professors F. D. Quigley and F. B. Wright for their guidance and encouragement during the preparation of the author's doctoral dissertation of which this paper is an expanded portion.

\section{REFERENCES}

1. S. K. Berberian, The regular ring of a finite $A W^{*}$-algebra, Ann. of Math. (2) 65 (1957), 224-240.

2. Note on a theorem of Fuglede and Putnam, Proc. Amer. Math. Soc. 10 (1959), 175-182.

3. H. F. Bohnenblust and S. Kakutani, Concrete representation of $(M)$-spaces, Ann. of Math.

(2) 42 (1941), 1025-1028.

4. M. M. Day, Normed linear spaces, Ergebnisse der Mathematik und ihrer Grenzgebiete, Springer, Berlin, 1958.

5. J. Dixmier, Les algèbres d'opérateurs dans l'espace hilbertien (Algèbres de von Neumann), Gauthier-Villars, Paris, 1957.

6. __ Les fonctionnelles linéaires sur l'ensemble des opérateurs bornés d'un espace de Hilbert, Ann. of Math. (2) 51 (1950), 387-408.

7. M. Fukamiya, Y. Misonou and Z. Takeda, On order and commutativity of $B^{*}$-algebras, Tôhoku Math. J. 6 (1954), 89-93.

8. R. V. Kadison, Order properties of bounded self-adjoint operators, Proc. Amer. Math. Soc. 2 (1951), 505-510.

9. I. Kaplansky, $A$ theorem on rings of operators, Pacific J. Math. 1 (1951), 227-232.

10. T. Ogasawara, $A$ theorem on operator algebras, J. Hiroshima Univ. 18 (1955), 307-309.

11. S. Sherman, Order in operator algebras, Amer. J. Math. 73 (1951), 227-232.

12. M. H. Stone, Boundedness properties in function lattices, Canad. J. Math. 1 (1949), 176-186. 13. __ The generalized Weierstrass approximation theorem, Math. Mag. 21 (1948), 167-184, 237-254.

14. D. Topping, Vector lattices of self-adjoint operators, Thesis, Tulane University, New Orleans, La., 1962.

15. _ Vector lattices of self-adjoint operators, Bull. Amer. Math. Soc. 69 (1963), 251-255.

16. __ Vector lattices, Boolean rings and simple functions, Portugal. Math. (to appear).

\section{University OF Chicago,}

Chicago, Illinois 